\title{
Color Persistent Anisotropic Diffusion of Images
}

\author{
Freddie Åström, Michael Felsberg, and Reiner Lenz \\ Linköping University, SE-581 83 Linköping, Sweden \\ \{freddie.astrom,michael.felsberg\}@itn.liu.se, reiner.lenz@itn.liu.se
}

\begin{abstract}
Techniques from the theory of partial differential equations are often used to design filter methods that are locally adapted to the image structure. These techniques are usually used in the investigation of gray-value images. The extension to color images is non-trivial, where the choice of an appropriate color space is crucial. The RGB color space is often used although it is known that the space of human color perception is best described in terms of non-euclidean geometry, which is fundamentally different from the structure of the RGB space. Instead of the standard RGB space, we use a simple color transformation based on the theory of finite groups. It is shown that this transformation reduces the color artifacts originating from the diffusion processes on RGB images. The developed algorithm is evaluated on a set of real-world images, and it is shown that our approach exhibits fewer color artifacts compared to state-of-the-art techniques. Also, our approach preserves details in the image for a larger number of iterations.
\end{abstract}

Keywords: non-linear diffusion, color image processing, perceptual image quality.

\section{Introduction}

In this paper we consider the problem of anisotropic diffusion of color images. By decorrelating the RGB color space we derive an improved color diffusion scheme which exhibits fewer color artifacts compared to state-of-the-art techniques. Moreover, using different edge-stopping functions for each decorrelated channel yields a diffusion tensor that does not effect structures in other color channels.

Diffusion filtering is based on partial differential equations (PDEs). In image enhancement applications a diffusion scheme is called anisotropic if the filterprocess is adapted according to the image structure. On the other hand, if the diffusion scheme does not take into account the underlying structure, it is called isotropic diffusion. Perona and Malik [8] were first to propose a PDE-based anisotropic diffusion scheme

$$
\partial_{t} u=\operatorname{div}\left(g\left(|\nabla u|^{2}\right) \nabla u\right),
$$

with diffusivity function $g(|\nabla u|)=\left(1+(|\nabla u| / K)^{2}\right)^{-1}$ where $K$ is a contrast parameter and $\nabla u$ is the image gradient. In the terminology of Weickert [17], the

A. Heyden and F. Kahl (Eds.): SCIA 2011, LNCS 6688, pp. 262-272, 2011.

(C) Springer-Verlag Berlin Heidelberg 2011 
Perona-Malik diffusivity is non-linear scalar diffusion and anisotropic diffusion denotes methods that use a diffusion tensor

$$
D=\sum_{i} g\left(\lambda_{i}\right) \mathbf{e}_{i} \mathbf{e}_{i}^{T},
$$

where $\lambda_{i}$ are the eigenvalues and $\mathbf{e}_{i}$ the eigenvectors of the structure tensor [3]

$$
T=\int w\left(\begin{array}{ll}
\partial u_{x} \partial u_{x} & \partial u_{x} \partial u_{y} \\
\partial u_{y} \partial u_{x} & \partial u_{y} \partial u_{y}
\end{array}\right) d x d y .
$$

$w$ is typically a Gaussian weight function. The diffusion equation now takes the form

$$
\partial_{t} u=\operatorname{div}(D \nabla u)
$$

If the eigenvalues of the structure tensor is $\lambda_{i}=\lambda_{i+1}, \forall i$ the anisotropic diffusion equation reduces to isotropic (scalar) diffusion. The presented diffusion process is applicable for scalar (gray-valued) images [1, 10, 12].

\subsection{Related Work}

Several PDE based approaches to suppress noise in color images [2, 5, 15, 9, 18, 14, 13. have been suggested in the past. Despite the progress in the field of color PDE filtering, the most frequently occurring drawback of the existing methods is the generation of color artifacts near edges. This problem will be referred to as a lack of color persistency. The reason for this type of artifact is that an edge (or noise) in one channel may not be present in the same location in another channel.

A relatively straightforward extension from gray image diffusion to color image diffusion is to use the same diffusion tensor for each of the color channels. This standard approach is described by Weickert [18 where the diffusion tensor is computed from the weighted average sum of the structure tensors for each RGB channel. An alternative approach was taken by Tang et al. [14] who map the RGB color vector space to two separate components, the direction (chromaticity) and the magnitude (brightness). Thereafter, they perform the diffusion process separately on these two channels. An important point made in their work is filtering the chromaticity channel can introduce color artifacts. Sochen et. al [13, have perhaps taken the most general approach to deriving the diffusion process. They view a two-dimensional (2D) image as a surface in 3D space and a color image as $2 \mathrm{D}$ surfaces in a $5 \mathrm{D}$ space. According to these mappings they define a metric and derive what they call the Beltrami flow.

\subsection{Main Contribution}

In this work we propose a color diffusion method based on a PCA-like technique to decorrelate the RGB color space, derived by Lenz and Carmona [7. Furthermore, we compare our diffusion approach with the standard approach described in 18 on real-world natural images and standard test images. Also, 
the proposed diffusion scheme is compared to the color space derived from decorrelating the RGB channels using standard principal component analysis and the Beltrami flow [13. The experiments show that our approach is in general more color persistent in comparison with these state-of-the-art techniques.

\section{Methods}

\subsection{Standard Approach to Color Image Diffusion}

What we call the standard approach is to sum the structure tensors of the RGB channels [18]

$$
T=\sum_{i} w_{i} T_{i}=\sum_{i} w_{i} \sum_{j} \lambda_{i j} \mathbf{e}_{i j} \mathbf{e}_{i j}^{T}
$$

where $i$ is the indices of the color channels and $\sum_{i} w_{i}=1$ is a weight. The diffusion function used in this work to compute the diffusion tensor is $g(\lambda)=$ $\exp (-\lambda / K)$ [4].

\subsection{Color Model}

The RGB color space channels are correlated [11. However, the noise that may exist in an image may not be correlated across channels. This can introduce color artifacts if the RGB space is not decorrelated prior to applying the diffusion filtering process. In an attempt to decorrelate the R, G and B channels, Lenz and Carmona [7] introduced the transformation matrix

$$
P=\frac{1}{\sqrt{3}}\left(\begin{array}{ccc}
1 & 1 & 1 \\
\sqrt{2} & \sqrt{2} \cos (2 \pi / 3) & \sqrt{2} \cos (4 \pi / 3) \\
0 & \sqrt{2} \sin (2 \pi / 3) & \sqrt{2} \sin (4 \pi / 3)
\end{array}\right) .
$$

This transformation is derived from the assumption that permutations of the three RGB channels are on average equally probable. Using tools from the representation theory of the permutation group $\mathrm{S}(3)$ it can be shown that the result is a decorrelation of the original RGB variables into a one-dimensional intensity, $I$, and a two-dimensional color-opponent component. In cases where the assumption of equally probable permutations is satisfied it can be shown that the rows of this matrix are the eigenvectors of the correlation matrix computed from the RGB vectors and that the color-opponent components belong to a two-dimensional eigenspace belonging to the same eigenvalue.

\subsection{New Approach to Color Image Diffusion}

In this work we propose a new approach to color image diffusion, with the aim to reduce color artifacts. The proposition is to first transform the RGB color space 
onto the irreducible representation (6) and to compute the diffusion tensors $D_{1}$, $D_{2}$ and $D_{3}$, one for each component in the transformed color space

$$
D=\left(\begin{array}{ccc}
D_{1} & 0 & 0 \\
0 & D_{2} & 0 \\
0 & 0 & D_{3}
\end{array}\right)
$$

$D_{i}=\sum_{j} g_{i}\left(\lambda_{i j}\right) \mathbf{e}_{i j} \mathbf{e}_{i j}^{T}$ allows for different diffusivity functions $g_{i}(\cdot)$ to be used in different channels. However, in order to enable a comparative evaluation of the two filtering techniques, the diffusion function described in section 2.1 will be used to compute all diffusion tensors, although with different contrast factors K.

\section{Experiments}

\subsection{Data Set}

To evaluate the two approaches to color diffusion, the algorithms are applied to real-world RGB color images obtained from a data set of road signs. The test images have been used with the permission of the authors of [6]. The images were taken in an urban environment. The camera was mounted in the cabin of a car behind the windscreen. Appropriate regions were cropped from the full images $(1280 \times 960$ pixels $)$ which are of JPEG format. Hence, color artifacts introduced by the JPEG-compression are already present in the images. Regions of interest are edges with different color tone and intensity. Images image00756.jpg and image00312.jpg from the data set were used to extract regions $\mathrm{S} 1$ and $\mathrm{S} 2$ of size $512 \times 512$ pixels seen in Fig. 3] and Fig. 4. Regions of size $56 \times 56$ pixels were zoomed in S1 and S2 seen in the same figures. Standard test images used are the Mandrill and Lena $(512 \times 512$ pixels $)$ seen in Fig. 11 and Fig. 2, Regions of interest of size $56 \times 56$ pixels were extracted from both images, in order to illustrate color artifacts.

\subsection{Tested Methods}

Four diffusion filtering methods are compared with respect to color persistency. The first method is the standard approach where the structure tensor is summed and weighted equally. The second, proposed approach, is based on decorrelating the standard RGB color space using (6) prior to computing the structure tensors. The diffusion processes were implemented using a finite-difference approximation and yield the iterative update scheme

$$
u_{t+1}=u_{t}+\tau\left(\nabla D \nabla u_{t}+D \nabla^{2} u_{t}\right),
$$

where the index $t$ is current iteration. $\tau$ is a step parameter and should not be selected too large, as the diffusion process will then violate the scale-space properties stated in [17, for a stable diffusion process as $t \rightarrow \infty$. The stopping time 
$t_{\mathrm{MAx}}$ was determined such that all algorithms terminated when a negative slope of their error curve was observed. The negative exponential diffusion function described in section 2.1 was used for all components in all diffusion schemes. For the experiments, the contrast parameter $K$ was set to $10^{-3}$ in the standard approach and in the intensity channel $I$ of the proposed approach. For the two color balance channels in the new approach, $K$ was set to $10^{-1}$. The third method used is decorrelation of the RGB color space using standard principal component analysis (PCA). Eigenvectors are computed from the average of all test images to enable a fair comparison between the proposed method and the PCA decomposition. In this case the contrast parameter was set to $10^{-3}$. The final and fourth method, is the state-of-the-art Beltrami flow, which for images Baboon, S1 and S2 used a contrast parameter of $10^{-3}$ and for the Lena image we used $10^{-2}$. All parameter values were set empirically.

The step length was set as follows, in the Baboon image all implementations used steplength $10^{-1}$ except for the Beltrami flow which used $10^{-3}$. For the Lena image step length was set to $10^{-1}$ for all implementations. In images S1 and S2 all algorithms were set to use a step length of $1 / 5$ except the Beltrami flow, which used a steplength of $10^{-3}$.

\subsection{Performance Evaluation}

Since color artifacts are primarily introduced around edges, these regions are zoomed in order to visually get an understanding of the color distortion. Quantitative measurements are often given by the peak-signal-to-noise ratio (PSNR) in the literature. However, the structural similarity index (SSIM) has been shown to be a more accurate measurement when determining image similarities [16]. Parameters of the index were set to the default values as recommended by the author [16. The source code to compute the MSSIM is available online (http:// www.ece.uwaterloo.ca/ z70wang/research/ssim/). To apply the SSIM index to the RGB color space, we have chosen to apply the SSIM measure to each individual color component and average the result. This approach was chosen since there is, to be best of our knowledge, no previously published work on the effect of applying the SSIM measurement to individual color components in the RGB color space.

\section{Results}

The four diffusion filtering approaches were tested and compared as described in section 3.2. Figures are organized as follows: from left to right and up to down: original image and zoomed region, noisy image, output after maximum number of iterations of our approach, sum of tensors, PCA and finally the Beltrami flow. Each of the algorithms has a corresponding zoomed region to their right of the corresponding full image. First a perceptual analysis based on the visual impression of the filtering outputs will be done, thereafter an quantitative analysis is made based on the SSIM index. Figures are best viewed in color. 
Gaussian noise of zero mean and 0.01 standard-deviation has been applied to the Mandrill and Lena images seen in Fig. 1] and Fig. 2, A subjective assessment of the diffusion process is that as the number of iterations increase, high-frequency components in the Mandrill image become more blurred in the standard approach. Visual inspection reveal that the structure in the Mandrill's zoomed region is preserved longer before becoming blurred in our method compared to the standard approach. The result of the PCA approach is competitive with our proposed color space, but visual inspection of the zoomed regions in Fig. 1 show that color artifacts have been introduced in the high-frequency regions. The Beltrami flow cannot handle the noise level and does introduce color artifacts. A similar result is seen in Fig. 2, but after 200 iterations it is recognized that there is no perceptual difference between our proposed approach and diffusion in the decorrelated PCA color space. However, the standard approach experience excessive blurring not seen in any other method.
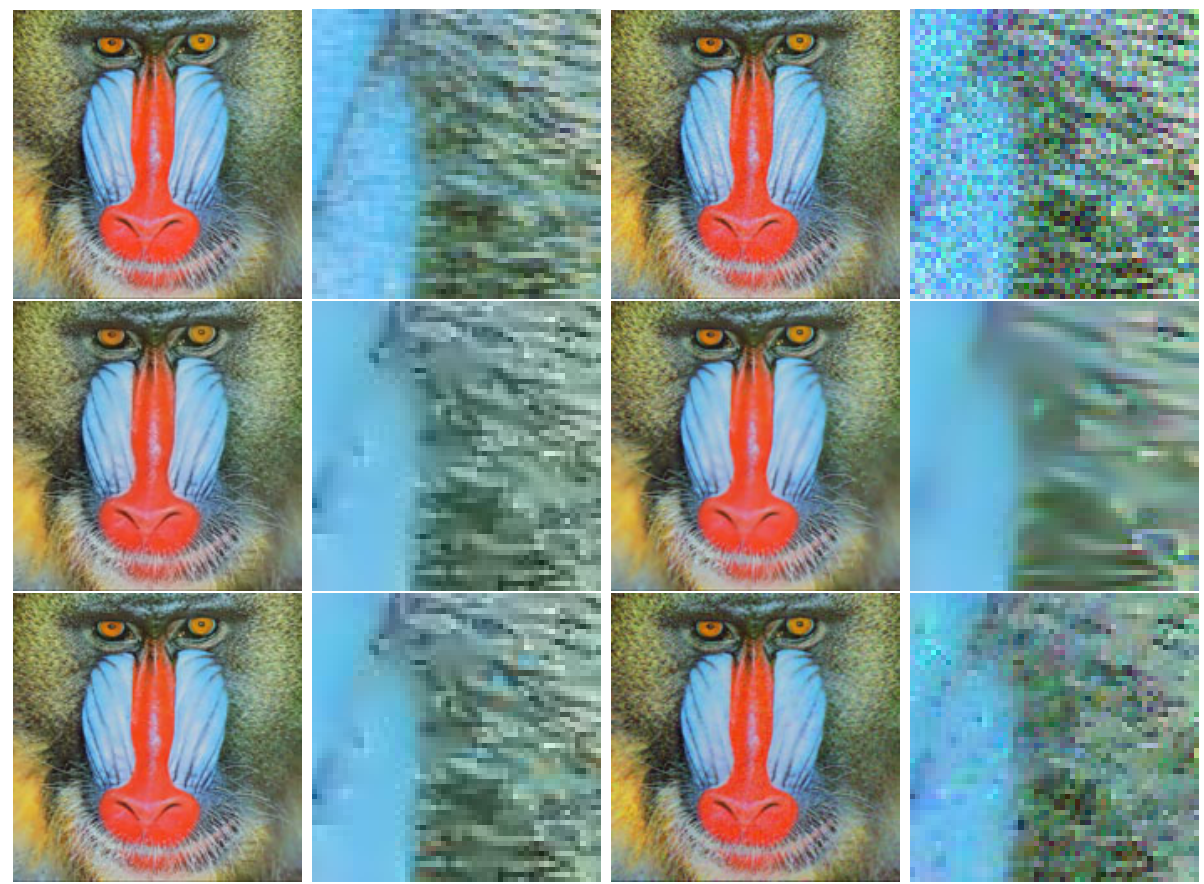

Fig. 1. Mandrill with zero mean and $\sigma=0.01$ Gaussian noise. Results are shown after 100 iterations. See text for details.

Image S1 is shown in Fig. 3, interesting parts of the zoomed region is the gable of the building. Total number of iterations for this image was set to 200 . In the standard approach and the Beltrami flow the white gable is severely blurred and color artifacts has been introduced around the frame of the window. Diffusion in our proposed color space preserves the gable and no color artifact is introduced 

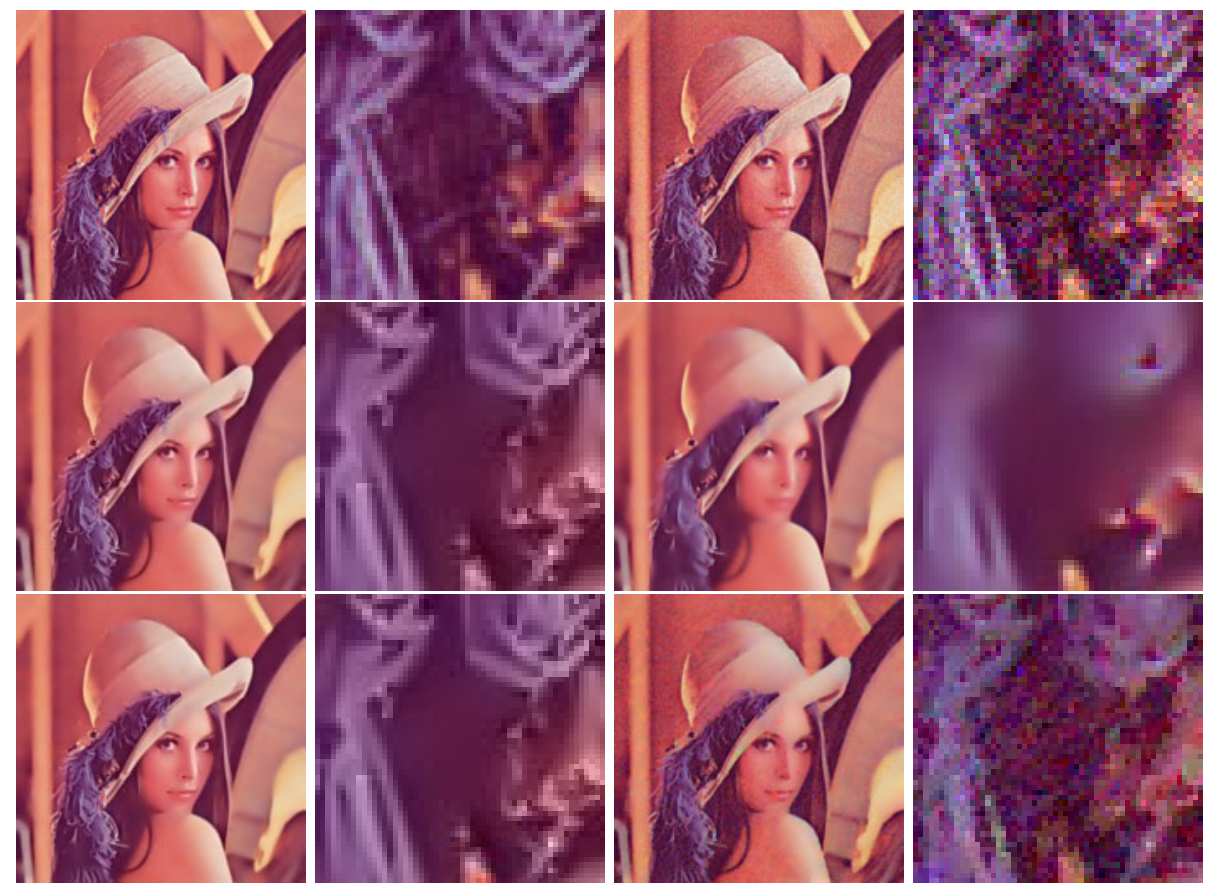

Fig. 2. Lena with zero mean and $\sigma=0.01$ Gaussian noise. Results are shown after 200 iterations. See text for details.

around the frame of the window as seen in the PCA color space. Image S2 is run for 100 iterations. It is seen in this test image that our method does perform slightly worse than the PCA on the edge of the road sign, but considering the trunk of the branch in the zoomed image a color artifact was introduced in the PCA not seen in our approach. Furthermore, the standard filtering and the Beltrami flow is considerably worse than the other two diffusion schemes.

A quantitative measurement of the two diffusion approaches was made based

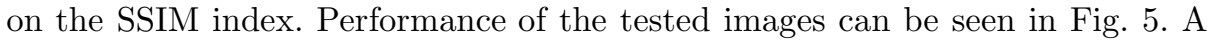
higher MSSIM value (maximum value is 1 ) indicate that the structure of the filtered image is more similar to the noise free image. Comparing the proposed and other methods in Fig. 5, it can be seen that the overall result for all the images are significantly better for the proposed filtering method.

An observation with regards to the Mandrill error graph is that for iterations 100 the PCA performs better than our proposed method. However, in Fig. 1 it is visible that, our approach produces perceptually more similar results to the ground truth compared to the PCA approach. Furthermore, considering the slope of the error curves, it is obvious that the standard approach and the Beltrami flow will continue to degrade the image quality to a larger extend than our method. 


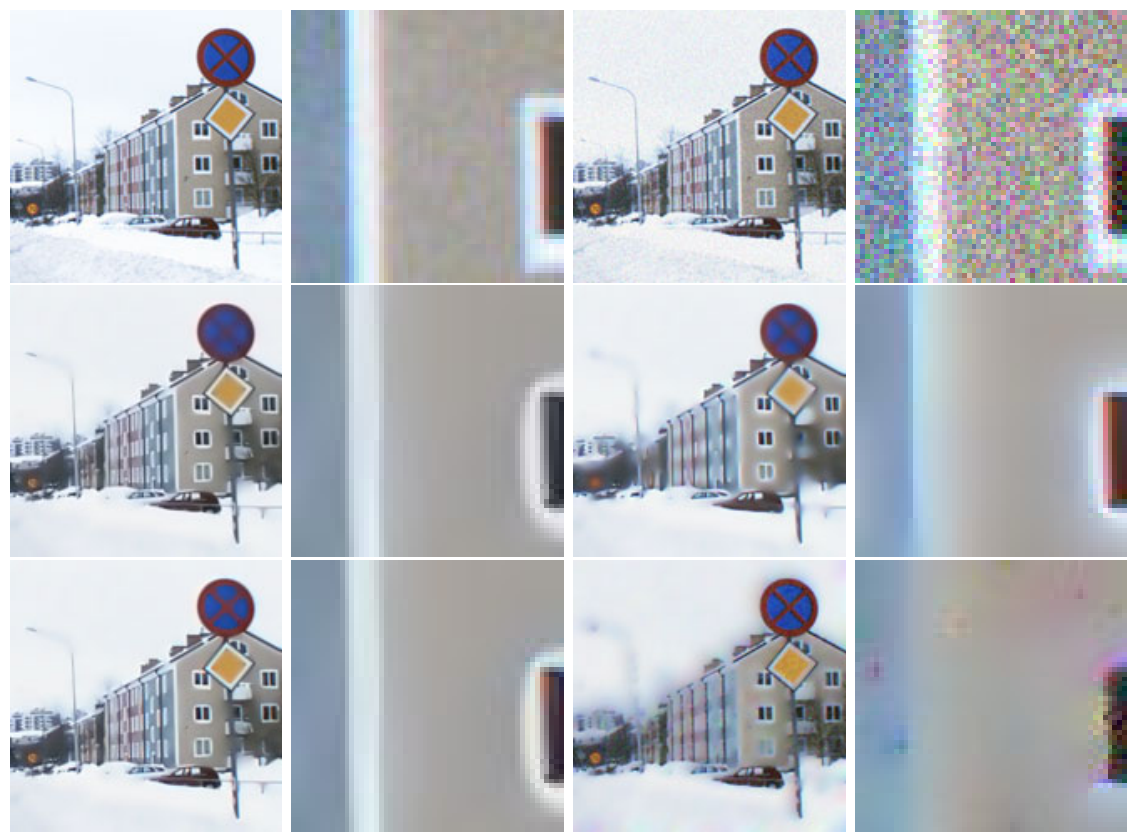

Fig. 3. S1 with zero mean and $\sigma=0.01$ Gaussian noise. Results are shown after 200 iterations. See text for details.

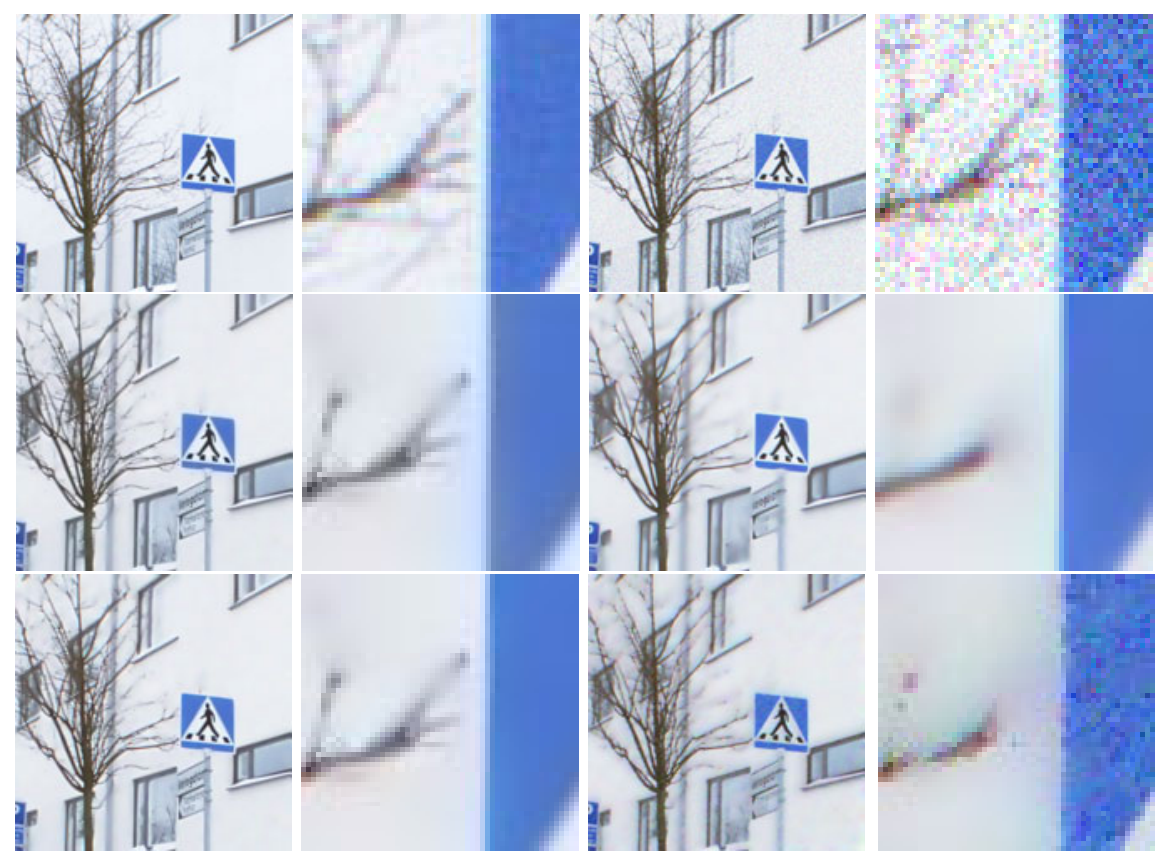

Fig. 4. S2 with zero mean and $\sigma=0.01$ Gaussian noise. Results are shown after 100 iterations. See text for details. 

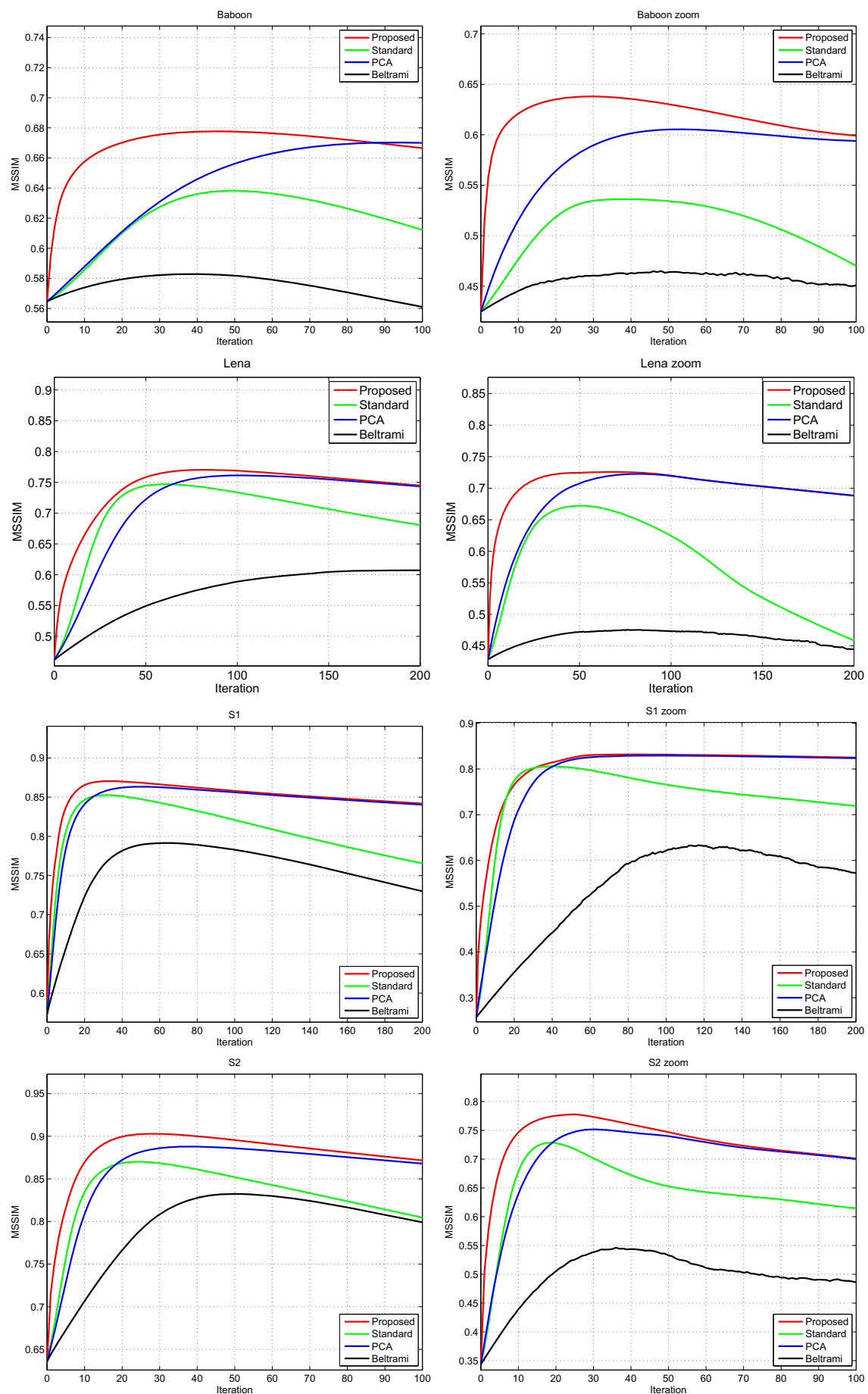

Fig. 5. MSSIM-values for proposed and implemented diffusion filtering methods 


\section{Conclusion}

In this work a novel color image diffusion method is introduced, where the standard euclidean RGB color space is transformed to a non-euclidean representation. The basis of the new color space represents the color vector intensity and color balance. It has been shown that non-linear diffusion in the proposed color space introduces fewer color artifacts compared to standard state-of-theart diffusion techniques. The findings are supported quantitatively by a higher structural similarity index.

\section{Acknowledgement}

This research has received funding from the The Swedish Research Council through a grant for the project Non-linear adaptive color image processing, from the EC's 7th Framework Programme (FP7/2007-2013), grant agreement 247947 (GARNICS), and by ELLIIT, the Strategic Area for ICT research, funded by the Swedish Government.

\section{References}

[1] Black, M.J., Sapiro, G., Marimont, D.H., Heeger, D.: Robust anisotropic diffusion. IEEE Transactions on Image Processing 7(3), 421-432 (1998)

[2] Chambolle, A.: Partial differential equations and image processing. In: Proceedings of the IEEE International Conference Image Processing, ICIP 1994, vol. 1, pp. 16-20 (November 1994)

[3] Felsberg, M.: On the relation between anisotropic diffusion and iterated adaptive filtering. In: Rigoll, G. (ed.) DAGM 2008. LNCS, vol. 5096, pp. 436-445. Springer, Heidelberg (2008)

[4] elation-Driven Diffusion Filtering. IEEE Transactions on Image Processing (2011), doi:10.1109/TIP.2011.2107330

[5] Kimmel, R., Malladi, R., Sochen, N.: Images as embedded maps and minimal surfaces: Movies, color, texture, and volumetric medical images. International Journal of Computer Vision 39, 111-129 (2000)

[6] Larsson, F., Felsberg, M., Forssen, P.E.: Patch contour matching by correlating fourier descriptors. In: Digital Image Computing: Techniques and Applications, DICTA 2009, pp. 40-46 (December 2009)

[7] Lenz, R., Carmona, P.L.: Hierarchical s(3)-coding of rgb histograms. In: Selected Papers from VISAPP 2009, vol. 68, pp. 188-200. Springer, Heidelberg (2010)

[8] Perona, P., Malik, J.: Scale-space and edge detection using anisotropic diffusion. IEEE Transactions on Pattern Analysis and Machine Intelligence 12, 629-639 (1990)

[9] Renner, A.I.: Anisotropic Diffusion in Riemannian Colour Space. Ph.D. thesis, Ruprecht-Kars-Universitt, Heidelberg (2003)

[10] Scharr, H., Black, M.J., Haussecker, H.W.: Image statistics and anisotropic diffusion. In: Proceedings of the Ninth IEEE International Conference on Computer Vision, pp. 840-847 (October 2003) 
[11] Sharma, G.: Digital Color Imaging Handbook. CRC Press, Inc., Boca Raton (2002)

[12] Sochen, N., Kimmel, R., Bruckstein, A.: Diffusions and confusions in signal and image processing. Journal of Mathematical Imaging and Vision 14, 195-209 (2001)

[13] Sochen, N., Kimmel, R., Malladi, R.: A general framework for low level vision. IEEE Transactions on Image Processing 7(3), 310-318 (1998)

[14] Tang, B., Sapiro, G., Caselles, V.: Color image enhancement via chromaticity diffusion. IEEE Transactions on Image Processing 10, 701-707 (2002)

[15] Tschumperle, D., Deriche, R.: Diffusion pdes on vector-valued images. IEEE Signal Processing Magazine 19(5), 16-25 (2002)

[16] Wang, Z., Bovik, A., Sheikh, H., Simoncelli, E.: Image quality assessment: from error visibility to structural similarity. IEEE Transactions on Image Processing 13(4), 600-612 (2004)

[17] Weickert, J.: Anisotropic diffusion in image processing (1996)

[18] Weickert, J.: Coherence-enhancing diffusion of colour images. Image and Vision Computing 17(3-4), 201-212 (1999) 